Marquette University

e-Publications@Marquette

\title{
Benzalkonium Chloride Alters Phenotypic and Genotypic Antibiotic Resistance Profiles in A Source Water Used for Drinking Water Treatment
}

Katherine R. Harrison

Marquette University

Anthony D. Kappell

Marquette University, anthony.kappell@marquette.edu

Patrick J. McNamara

Marquette University, patrick.mcnamara@marquette.edu

Follow this and additional works at: https://epublications.marquette.edu/civengin_fac

Part of the Civil Engineering Commons

\section{Recommended Citation}

Harrison, Katherine R.; Kappell, Anthony D.; and McNamara, Patrick J., "Benzalkonium Chloride Alters Phenotypic and Genotypic Antibiotic Resistance Profiles in A Source Water Used for Drinking Water Treatment" (2020). Civil and Environmental Engineering Faculty Research and Publications. 249.

https://epublications.marquette.edu/civengin_fac/249 
Marquette University

e-Publications@Marquette

\section{Civil, Construction and Environmental Engineering Faculty Research and Publications/College of Engineering}

This paper is NOT THE PUBLISHED VERSION; but the author's final, peer-reviewed manuscript. The published version may be accessed by following the link in the citation below.

Environmental Pollution, Vol. 257 (February 2020): 113472. DOI. This article is C Elsevier and permission has been granted for this version to appear in e-Publications@Marquette. Elsevier does not grant permission for this article to be further copied/distributed or hosted elsewhere without the express permission from Elsevier.

\section{Benzalkonium Chloride Alters Phenotypic and Genotypic Antibiotic Resistance Profiles in A Source Water Used for Drinking Water Treatment}

Katherine R. Harrison

Civil, Construction and Environmental Engineering, Marquette University, Milwaukee, WI Anthony D. Kappell

Civil, Construction and Environmental Engineering, Marquette University, Milwaukee, WI Patrick J. McNamara

Civil, Construction and Environmental Engineering, Marquette University, Milwaukee, WI

\section{Highlights}

- Benzalkonium chloride (BAC) altered the antibiotic resistance profiles of bacteria in surface water. 
-BAC positively selected for bacteria resistant to ciprofloxacin and sulfamethoxazole.

-BAC selected against bacteria resistant to cephalexin, chloramphenicol, and streptomycin.

- Exposure to BAC over time showed increased relative abundance of the resistance genes sul1 and blaTEM.

\section{Abstract}

Antibiotic resistance is a major public health concern. Triclosan is an antimicrobial compound with direct links to antibiotic resistance that was widely used in soaps in the U.S. until its ban by the U.S. Food and Drug Administration. Benzalkonium chloride (BAC), a quaternary ammonium compound, has widely replaced triclosan in soaps marketed as an antibacterial. BAC has been detected in surface waters and its presence will likely increase following increased use in soap products. The objective of this study was to determine the effect of BAC on relative abundance of antibiotic resistance in a bacterial community from a surface water used as a source for drinking water treatment. Bench-scale microcosm experiments were conducted with microbial communities amended with BAC at concentrations ranging from $0.1 \mu \mathrm{g} \mathrm{L}-1$ to $500 \mu \mathrm{g} \mathrm{L}^{-1}$. Phenotypic antibiotic resistance was quantified by culturing bacteria in the presence of different antibiotics, and genotypic resistance was determined using qPCR to quantify antibiotic resistance genes (ARGs). BAC at concentrations ranging from 0.1 $\mu \mathrm{g} \mathrm{L^{-1 }}$ to $500 \mu \mathrm{g} \mathrm{L^{-1 }}$ was found to positively select for bacteria resistant to ciprofloxacin and sulfamethoxazole, and negatively select against bacteria with resistance to six other antibiotics. Exposure to BAC for 14 days increased the relative abundance of sul 1 and bla TEM. This study rehighlights the importance of employing both culture and non-culture-based techniques to identify selection for antibiotic resistance. The widespread use of BAC will likely impact antibiotic resistance profiles of bacteria in the environment, including in source waters used for drinking water, wastewater treatment plants, and natural waterways.

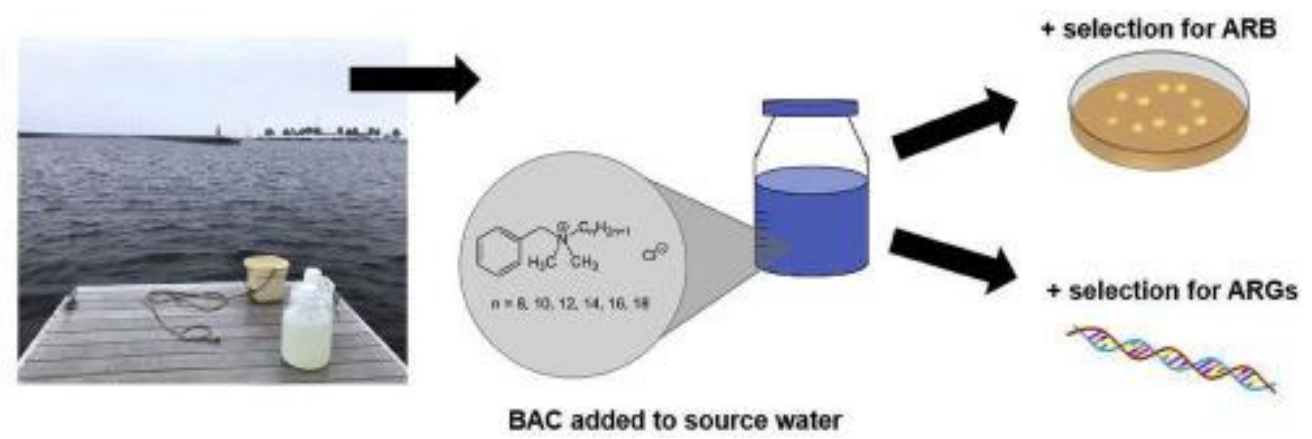

Keywords

Antimicrobial, Quaternary ammonium compounds, Triclosan, Antibiotic resistant bacteria

\section{Introduction}

Antibiotic resistance is a global health problem, and the World Health Organization warns that we could soon enter a post-antibiotic era (World Health Organization, 2015). In the United States, over 23,000 people die each year from antibiotic resistant infections, and treating these infections costs an estimated \$1 billion per year (US Dept. of Health and Human Services, 2013). An estimated 10 million 
people could die from antibiotic resistant infections globally by 2050 if no action is taken to reduce the spread of antibiotic resistance (O'Neill, 2016). Since the proliferation of antibiotic resistance in the environment could increase global antibiotic resistance that impacts public health, it is important to understand what chemicals can potentially selecte for antibiotic resistance and how to mitigate their effects.

Many chemicals apply selective pressure that can select for antibiotic resistance in the environment (Gadea et al., 2017; Jutkina et al., 2018; Zhang et al., 2017, 2018). Antibiotics present a paradox whereby the more we use them the less effective they become because bacteria continue to acquire resistance (Barber, 2019). The use of antibiotics for medicinal and agricultural purposes leads to increases in antibiotic resistance (Berendonk et al., 2015). Synthetic antimicrobial compounds used for cleaning purposes have also been linked to antibiotic resistance (Tezel and Pavlostathis, 2015). Triclosan, an antimicrobial, was widely used in hand soaps and is still used in toothpastes in the U.S. and globally. Triclosan has been linked to increased tolerance to antibiotics and antibiotic resistance genes (ARGs) in both pure culture studies and mixed microbial studies (Carey et al., 2016b; Carey and McNamara, 2015, 2016; Liu and Han, 2008; McMurry, Oethinger, and Levy, 1998). Triclocarban is another antimicrobial compound that was previously found to be in even higher abundance than triclosan in U.S. biosolids (McClellan and Halden, 2010) and linked to selection for antibiotic resistance (Carey et al., 2016a; Carey and McNamara, 2015) The link of triclosan and triclocarban to antibiotic resistance along with their lack of benefit when added to hand soaps resulted in their ban from overthe-counter soaps in the U.S by the U.S. Food and Drug Administration (FDA) in 2016 (McNamara and Levy, 2016).

Benzalkonium chloride (BAC) is an antimicrobial agent that was not included in the recent ban by the U.S. FDA and is now widely used in hand soaps. BAC belongs to the class of quaternary ammonium compounds which are often used as surfactants. BAC can be comprised of many different alkylbenzyldimethylammonium chlorides with variable even-numbered alkyl carbon chain lengths within the mixture. Exposure to BAC has been linked to increased selection for antibiotic resistance and co-resistance to biocides and metals (Pal et al., 2015). For example, aerobic sediment communities exposed to BAC had increased phenotypic resistance to ciprofloxacin (Tandukar et al., 2013). BAC inevitably ends up in surface waters because it is a major consumer product (Clara et al. 2007a, b; Zhang et al., 2015) with the potential to enter drinking water treatment plants. Research on antibiotic resistance in drinking water treatment plants and their distribution systems has been increasing because of the direct exposure route of antibiotic resistance from drinking water to people (Garner et al., 2018; Kappell et al., 2019; Xi et al., 2009; Xu et al., 2016) It is likely that BAC could impact the antibiotic resistance profiles of bacteria and their genes in surface waters used for drinking water treatment. Research is not available to describe how BAC alters antibiotic resistance in a source water used for drinking water.

The objective of this research was to determine if BAC selects for antibiotic resistant bacteria and resistance genes in a source water used for drinking water treatment. Based on previous sediment research (Tandukar et al., 2013), it was hypothesized that BAC would increase phenotypic resistance to ciprofloxacin, an important antibiotic for public health purposes. A surface water sample was collected from Lake Michigan, a body of water used for several drinking water utilities, and amended with 
varying levels of BAC. Phenotypic resistance to antibiotics was assessed through culture-based plating techniques, and genotypic resistance was assessed through non-culture-based qPCR. This is the first research to present information on the impacts of BAC on antibiotic resistance profiles in a source water used for drinking water. These results provide insights into the possible ramifications of widespread presence of BAC in the environment if it is to be used as widely as triclosan and triclocarban were used.

\section{Materials and methods}

\subsection{Reagents and supplies}

A $50 \% \mathrm{w} / \mathrm{w}$ aqueous solution of BAC was manufactured by Alfa Aesar ${ }^{\mathrm{TM}}$ (Ward Hill, United States). The BAC mix was comprised of benzyl-C8-18-alkyldimethyl chlorides from C8 to C18 carbon chain lengths. Product specifications indicate water content to be $>48.0$ to $<52.0 \%$ (Cat No. AAB20760AP, Mfr No. B20760AP). Ampicillin sodium salt (VWR, Cat No. 97061-442) was dissolved in Milli-Q ${ }^{\circledR}$ water at $32 \mathrm{mg} \mathrm{mL}^{-1}$ and filter sterilized. Chloramphenicol $\geq 98 \%$ (Sigma Life Science, Product No. C0378) was dissolved in $100 \%$ ethanol at $32 \mathrm{mg} \mathrm{mL}^{-1}$. Sulfamethoxazole (Sigma-Aldrich, Product No. S7507) was dissolved in acetone at $100 \mathrm{mg} \mathrm{mL}^{-1}$. Erythromycin (Sigma-Aldrich, Product No. E6376) was dissolved in $100 \%$ ethanol at $8 \mathrm{mg} \mathrm{mL}^{-1}$. Streptomycin sulfate salt (Sigma-Aldrich, Product No. S6501) was dissolved in Milli-Q ${ }^{\circledR}$ water at $64 \mathrm{mg} \mathrm{mL}^{-1}$ and filter sterilized. Rifampicin (TCl America, Product No. R0079) was dissolved in dimethyl sulfoxide at $4 \mathrm{mg} \mathrm{mL}^{-1}$. Ciprofloxacin $\geq 98.0 \%$ (Sigma-Aldrich, Product No. 17850) was dissolved in Milli- ${ }^{\circledR}$ water and one drop $5 \mathrm{~N}$ hydrochloric acid at $4 \mathrm{mg} \mathrm{mL}^{-1}$ and filter sterilized. Trimethoprim (TCl America, Product No. T2286) was dissolved in dimethyl sulfoxide at $16 \mathrm{mg} \mathrm{mL}^{-1}$. Tetracycline $\mathrm{HCl}$ (Research Products International, Cat No. T17000) was dissolved in Milli-Q ${ }^{\circledR}$ water at $10 \mathrm{mg} \mathrm{mL}^{-1}$ and filter sterilized. Cephalexin monohydrate (Research Products International, Cat No. C59000) was dissolved in Milli- $\mathrm{Q}^{\circledR}$ water at $8 \mathrm{mg} \mathrm{mL}^{-1}$ and filter sterilized. Ultra-pure grade cycloheximide crystalline was obtained from VWR International (Cat No. 97064-724) and prepared in Milli- $Q^{\circledR}$ water at $100 \mathrm{mg} \mathrm{mL}^{-1}$ and filter sterilized. Dimethyl sulfoxide $\geq 99.9 \%$ was obtained from Sigma-Aldrich (Product No. 472301). 100\% ethanol was obtained from VWR International (KOPTEC, Cat No. 89125-186). HPLC grade acetone was obtained from Fisher Scientific (Fisher Chemical, Cat No. A949-1). Molecular genetics grade agar powder was purchased from Fisher Scientific (Fisher BioReagents ${ }^{\mathrm{TM}}$, Cat No. BP1423-500). R2A Broth for heterotrophic bacteria was purchased from VWR (HiMedia ${ }^{\circledR}$, Cat No. 95039-342).

\subsection{Microcosm setup}

Microcosms were used to analyze the effect of BAC on the phenotypic and genotypic resistance of the bacterial community. Water was sampled from the outer harbor of Lake Michigan in Milwaukee, Wisconsin and aseptically aliquoted into $1 \mathrm{~L}$ sterile clear glass bottles with $125 \mathrm{~mL}$ headspace. Bottles were amended with BAC at concentrations of $0,0.1,1.0,10,100$, or $500 \mu \mathrm{g} \mathrm{L}^{-1}$. Concentrations were based on $B A C$ concentrations present in the environment in surface waters $\left(1.9 \mu \mathrm{g} \mathrm{L}^{-1}\right)$, wastewater treatment influent $\left(0.810-170 \mu \mathrm{g} \mathrm{L}{ }^{-1}\right)$, wastewater treatment effluent $\left(0.014-0.630 \mu \mathrm{g} \mathrm{L}{ }^{-1}\right)$, hospital effluent ( $4000 \mathrm{~g} \mathrm{~L}^{-1}$ ), and known bacterial minimum inhibitory concentrations (MIC's) to BAC (40012,000 $\mathrm{g} \mathrm{L} \mathrm{L}^{-1}$ ) (Clara et al. 2007a, b; Lambert et al., 2001; Joynson et al., 2002; Loughlin et al., 2002; Martínez-Carballo et al., 2007). BAC is currently an active ingredient in many anti-bacterial consumer products at a concentration of $1.3 \times 10^{6} \mu \mathrm{g} \mathrm{L}^{-1}$ and becomes diluted in the wastewater 
treatment train and subsequent processes. The control community was not amended with BAC. BAC was added by diluting with Milli- $\mathrm{Q}^{\circledR}$ water as a vehicle for the chemical compound. Each microcosm treatment condition was tested in triplicate $(n=3)$. Bottles were loaded onto an orbital mixer at $125 \mathrm{rpm}$ to suspend cells and ensure continuous mixing. The bottles were kept at room temperature $\left(20 \pm 2.0^{\circ} \mathrm{C}\right.$ ) and covered to mitigate light and the growth of phototrophs (Wang et al., 2012). Agar plates were prepared with 10 distinct antibiotics. Water samples from each microcosm bottle were directly plated onto the agar plates on days $0,3,7$, and 14 with and without antibiotics to quantify phenotypic antibiotic resistance. For day 0 , water samples were aliquoted into three sterile $1 \mathrm{~L}$ bottles and directly plated on the same day with no treatment added. Water from source water (day 0 ) and remaining volume of microcosms from day 14 were filtered, extracted for DNA, and analyzed for ARGs via qPCR.

\subsection{Quantification of antibiotic resistant heterotrophic bacteria}

The quantification of total and antibiotic resistant heterotrophic bacteria was determined by direct plating onto R2A growth media with or without antibiotics and with $100 \mu \mathrm{g} \mathrm{mL}^{-1}$ of cycloheximide to inhibit fungal growth. Antibiotic concentrations included: ampicillin (AMP; $32 \mu \mathrm{gL}^{-1}$ ), chloramphenicol ( $\mathrm{CHL} ; 32 \mu \mathrm{g} \mathrm{mL} \mathrm{L}^{-1}$ ), sulfamethoxazole (SULF; $100 \mu \mathrm{g} \mathrm{mL} \mathrm{L}^{-1}$ ), erythromycin (ERY; $8 \mu \mathrm{g} \mathrm{mL}{ }^{-1}$ ), streptomycin (STREP; $64 \mu \mathrm{g} \mathrm{mL} \mathrm{L}^{-1}$ ), rifampicin (RIF; $4 \mu \mathrm{g} \mathrm{mL} \mathrm{L}^{-1}$ ), ciprofloxacin (CIP; $4 \mu \mathrm{g} \mathrm{mL}^{-1}$ ), trimethoprim (TRIM; $16 \mu \mathrm{g} \mathrm{mL}^{-1}$ ), tetracycline (TET; $16 \mu \mathrm{g} \mathrm{mL}^{-1}$ ), and cephalexin (CEPH; $8 \mu \mathrm{g} \mathrm{mL}{ }^{-1}$ ). The implementation of these agar plates with different antibiotics is a well-established method in our lab (Kappell et al., 2019) and in literature (Nagy et al., 2015; Watkinson et al., 2007). Concentrations were diagnostic concentrations inferring failure of treatment or clinical resistance based on CLSI dilution method (CLSI, 2018). A variety of antibiotics were used to represent various classes and uses which are pertinent to human health, although some of the most widespread antibiotics found in the environment are erythromycin, streptomycin, and trimethoprim (Gothwal and Shashidhar, 2015). A table listing the various classes of antibiotics tested is provided in Supplemental Table S2. Samples were spread plated onto agar media prior to incubation at $30{ }^{\circ} \mathrm{C}$ for 5 days. Based on resulting counts, water samples were diluted at $10^{\circ}, 10^{-1}, 10^{-2}, 10^{-3}, 10^{-4}$, and $10^{-5}$ and adjusted accordingly in order to yield countable (30-300) results. Counts were performed manually (Braoudaki and Hilton, 2004).

\subsection{DNA extraction}

DNA was extracted from filters used to capture DNA in the experiments. Specifically, water from all microcosms were harvested on day $14(615-1000 \mathrm{~mL})$ and aseptically quantified using a sterile clear glass graduated cylinder. The water was aseptically filtered through $0.2-\mu \mathrm{m}$ polycarbonate filters, and the filters were stored in sterile $2 \mathrm{~mL}$ tubes at $-20^{\circ} \mathrm{C}$ prior to extraction. In addition, three $1 \mathrm{~L}$ samples were harvested on day 0 and filtered onto $0.2-\mu \mathrm{m}$ polycarbonate filters, and the filters were stored at $-20^{\circ} \mathrm{C}$ prior to extraction. DNA extractions on the filters were performed per manufacturer instructions with the FastDNA Spin Kit (MP Biomedicals LCC, Solon, OH). Filters were initially broken by grinding with sterile pipet tips after tubes were exposed to liquid nitrogen. After addition of CLS-TC buffer, initial cell lysis was performed by subjecting the filters to three freeze-thaw cycles with liquid nitrogen followed by incubation at room temperature for $30 \mathrm{~min}$ (Kappell et al., 2019; McNamara et al., 2014). The filters were subjected to freeze-thaw cycles to ensure sufficient cell breakage. DNA yield was quantified by spectrophometric analysis with Nanodrop. 


\subsection{Quantification of antibiotic resistance genes}

Quantitative polymerase chain reactions (qPCR) were performed using a LightCycler 96 (Roche Applied Science). All reactions were a total volume of $20 \mu \mathrm{L}$ and utilized $1 \mathrm{X}$ PowerUp ${ }^{\mathrm{TM}} \mathrm{SYBR}^{\circledR}$ Green Master Mix (Applied Biosystems, Cat No. A25741), primers at a final concentration of $1.0 \mu \mathrm{M}$ each, and $5 \mu \mathrm{L}$ DNA diluted 1:50 to minimize inhibition in the optimal range for the target gene standard curve. All qPCR were performed under the following conditions: preincubation $\left(50^{\circ} \mathrm{C}, 120 \mathrm{~s}\right)$, preincubation and initial denaturation $\left(95^{\circ} \mathrm{C}, 600 \mathrm{~s}\right)$, three-step amplification $\left(95^{\circ} \mathrm{C}\right.$, 30s; annealing temperature specific to target gene, $30 \mathrm{~s} ; 72{ }^{\circ} \mathrm{C}, 30 \mathrm{~s} ; 40$ cycles), followed by melting curve analysis. The target genes

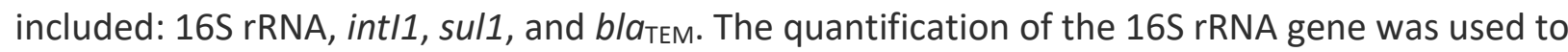
enumerate members of the domain Bacteria (LaPara et al., 2011). The integrase gene of the class 1 integron, intl1, was quantified because it is related to horizontal gene transfer and has been used as a proxy for anthropogenic pollution in the environment (Gillings et al., 2008, 2015). The sul1 gene confers resistance to sulfonamides, synthetic antimicrobial agents (Gao et al., 2012; Heuer et al., 2011; Pruden et al., 2006). Finally, the bla public health concern and many antibiotics including ampicillin and cephalexin belong to this category (Livermore, 1995; Poole, 2004). Each DNA sample was subjected to qPCR in triplicate and the copy numbers (CN) per L from the three wells were averaged. Acceptable $R^{2}$ values were greater than 0.98 and acceptable efficiency averages were greater than $84.5 \%$.

\subsection{Statistical analysis}

Statistical analysis was performed using the statistical package ' $R$ ' within 'R Studio' (Team $R$ Development Core, 2011). Significant differences between BAC treatment concentrations and time of exposure for absolute and relative abundance of antibiotic resistant bacteria (ARB) and ARGs was determined using one-way analysis of variance (ANOVA) with the 'aov' function. Post-hoc multiple pairwise comparisons were performed with Tukey's honestly significant differences (HSD) implemented with the 'TukeyHSD' function. Significant differences were defined as $p$-values less than 0.05 .

\section{Results and discussion}

\subsection{Impact of BAC on bacterial phenotypic selection for antibiotic resistance}

A source water was amended with varying concentrations of BAC and sampled over time. To assess impacts of BAC on antibiotic resistance, the phenotypic resistance profiles of BAC-amended communities were compared to the control community at the same time point (Fig. 1) and compared to themselves over time (Fig. 2). The relative abundance of bacteria resistant to ciprofloxacin was significantly greater when exposed to $0.1 \mu \mathrm{g} \mathrm{L}^{-1} \mathrm{BAC}$ by day 7 compared to the control with no exposure $(p=0.011)$. This finding is relevant because ciprofloxacin is currently used to treat a variety of bacterial infections, including severe cases of typhoid fever in children and in chronic typhoid carriers, and is a very important antibiotic for protection of public health (Ferreccio et al., 2019; Dutta et al., 1993). Ciprofloxacin was found to more frequently exceed safe levels in rivers globally than any other antibiotic tested in a recent international study (Wilkinson et al., 2019), and was the most abundant antibiotic found in a U.S. survey of biosolids (McClellan and Halden, 2010). The high prevalence of ciprofloxacin reflects its high usage rates, but has also triggered concerns due to its links in promoting cross-resistance. In a recent study with human-associated, wastewater-derived bacterial communities 
ciprofloxacin was often associated with multidrug resistance (Murray et al., 2019). Cross resistance to ciprofloxacin was also seen in anaerobic digester biomass exposed to triclosan (Carey and McNamara, 2016) corroborating the findings of this research that an antimicrobial, in this case BAC, has potential to lead to cross resistance to ciprofloxacin. Note that the relative abundance of bacteria resistant to ciprofloxacin in the BAC-amended communities were not different from the control at day 3 or day 14 ( $p>0.05$ ), indicating that selection for antibiotic resistance is transient (Kappell et al., 2019).
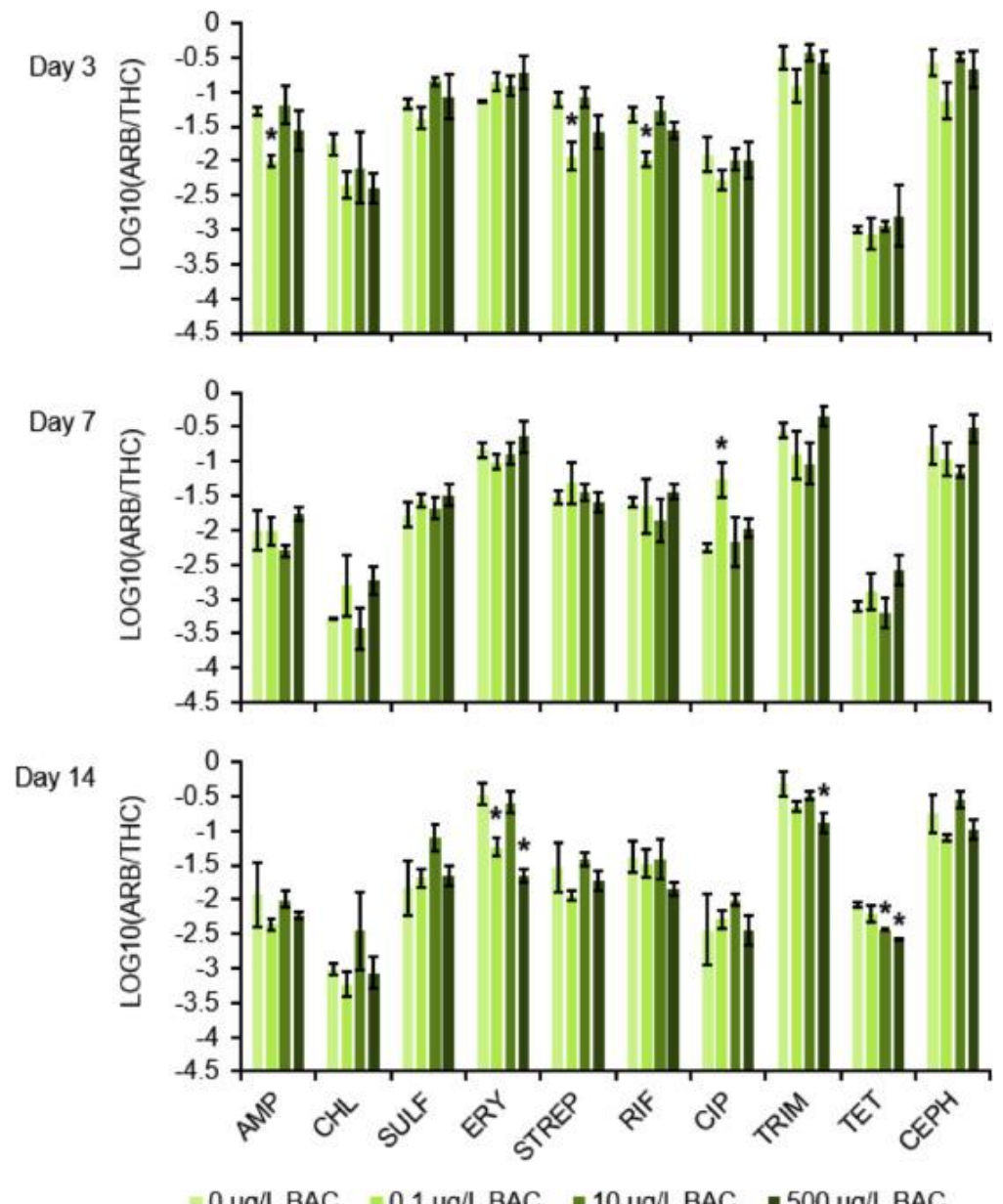

Fig. 1. Impact of $\operatorname{BAC}\left(0.1,10\right.$, and $\left.500 \mu \mathrm{g} \mathrm{L}^{-1}\right)$ relative to control $\left(0 \mu \mathrm{g} \mathrm{L}^{-1}\right)$ on phenotypic resistance to 10 antibiotics in the bacterial community of a source water for drinking water treatment. Bars represent average log-scaled relative abundance of antibiotic resistant bacteria (ARB) relative to total heterotrophic plate counts (THC) among three plates. A higher bar represents more bacterial colonies resistant to the associated antibiotic normalized to all bacterial colonies grown without the presence of an antibiotic. An * refers to a statistical increase or decrease $(p<0.05)$ compared to $0 \mu \mathrm{g} \mathrm{L} \mathrm{L}^{-1}$ BAC. Error bars represent standard deviation of the three relative abundances from ARB relative to $\operatorname{THC}(n=3)$. Total abundance including R2A counts are included in SI Fig. 1. 


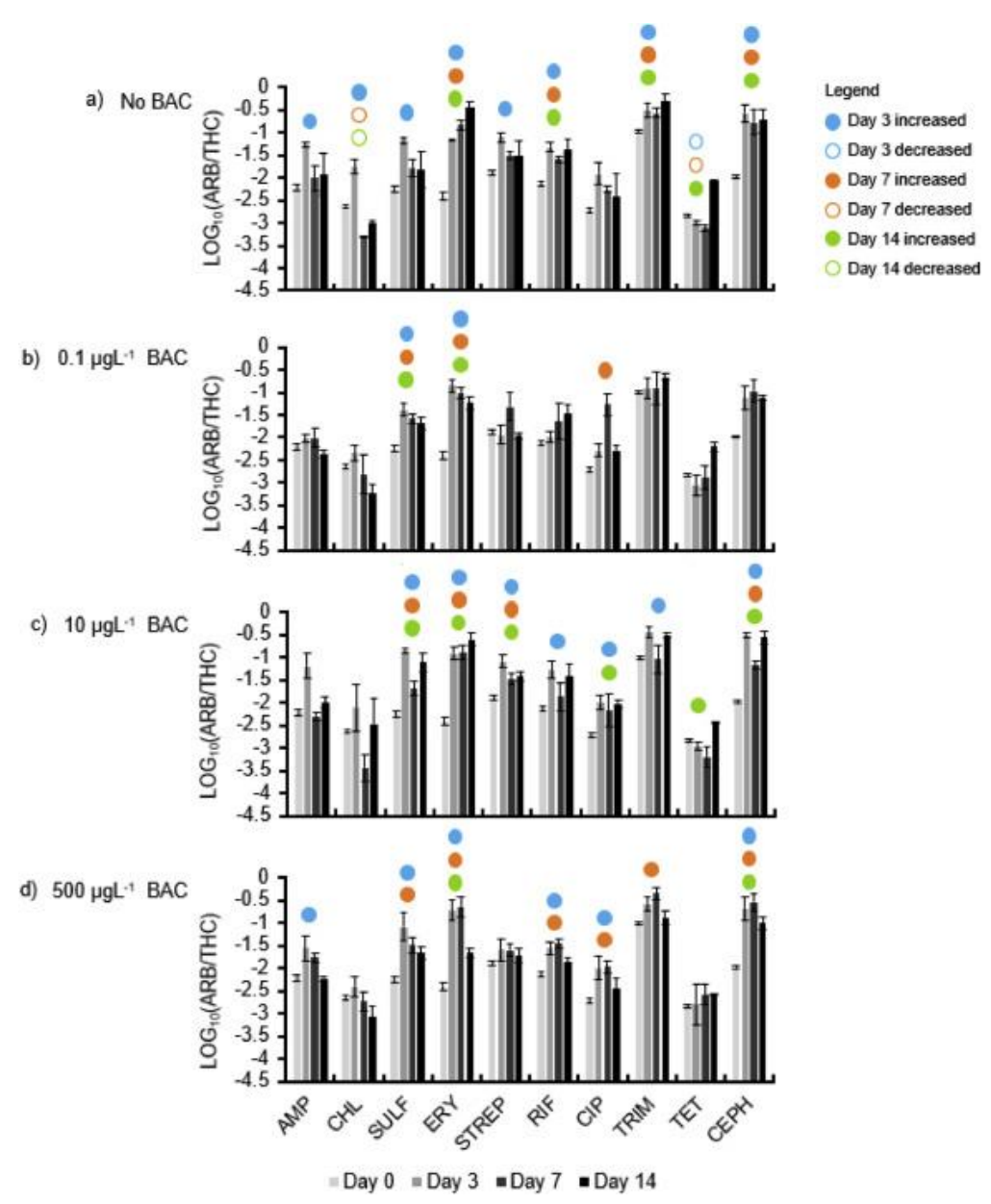

Fig. 2. Impacts of BAC on phenotypic resistance to 10 antibiotics in the bacterial community of a source water for drinking water treatment. Relative abundance of phenotypic resistance is shown over time relative to initial conditions (day 0) for the given concentration of BAC $\left(a=0 \mu \mathrm{g} \mathrm{L}^{-1}, b=0.1 \mu \mathrm{g} \mathrm{L}^{-1}, c=10 \mu \mathrm{g} \mathrm{L}^{-1}, \mathrm{~d}=500 \mu \mathrm{g} \mathrm{L}^{-1}\right.$ ). Relative abundance of antibiotic resistant bacteria (ARB) at day 0 is the same for all communities. Solid colored circles in legend refer to a statistical increase $(p<0.05)$ and open circles refer to a statistical decrease between the relative abundance of ARB on days 3, 7, and 14 compared to day 0 for the given BAC concentration. Bars represent average log-scaled relative abundance of ARB to total heterotrophic plate counts (THC) among three plates. A higher bar represents more bacterial colonies resistant to the associated antibiotic normalized to all bacterial colonies grown without the presence of an antibiotic. Error bars represent standard deviation of the three relative abundances from ARB relative to THC $(n=3)$.

Phenotypic resistance to ampicillin, streptomycin, and rifampicin of heterotrophic bacteria exposed to $0.1 \mu \mathrm{g} \mathrm{L} \mathrm{L}^{-1}$ BAC significantly decreased by day $3(p<0.031)$. Similarly, resistance to erythromycin, trimethoprim, and tetracycline of the community exposed to $500 \mathrm{gg} \mathrm{L}^{-1} \mathrm{BAC}$ significantly decreased by day $14(p<0.008)$. This finding is relevant because erythromycin, streptomycin, and trimethoprim are some of the most widespread antibiotics found in the environment (Gothwal and Shashidhar, 2015). Since BAC negatively selected for bacteria resistant to these antibiotics, it should be noted that BAC, at least in part, has potential to limit the proliferation of antibiotic resistance to a select few antibiotics at the concentrations tested. Erythromycin, streptomycin, and trimethoprim all treat various bacterial infections that are pertinent to human health and the impact BAC exposure has on a bacterial community used as a source water for drinking water treatment should be tested further to identify negative selection potential. 
Analysis of how the antibiotic resistance profiles changed over time for a given community reveal distinct trends in the control community and BAC-amended communities. Exposure to $0.1 \mu \mathrm{g} \mathrm{L}^{-1} \mathrm{BAC}$ led to increased abundance of phenotypic resistance to sulfamethoxazole and ciprofloxacin (Fig. 2b). The relative abundance of bacteria resistant to sulfamethoxazole increased by day 3 for both communities $(p<0.005)$, but the relative abundance of ARB to sulfamethoxazole only significantly increased on day 7 and day 14 compared to day 0 in the community treated with $0.1 \mu \mathrm{g} \mathrm{L}^{-1} \mathrm{BAC}$ $(p<0.006)$. These increases were not observed for the control community on day 7 and $14(p>0.242)$. Relative abundance of ARB at day 0 is the same for both communities. The relative abundance of ARB significantly increased by day 7 for ciprofloxacin when the community was treated with $0.1 \mu \mathrm{L} \mathrm{L}^{-1} \mathrm{BAC}$ $(p<0.001)$. No significant increases to ciprofloxacin were observed for any days in the control community $(p>0.999)$. Overall, addition of BAC showed potential for more increases in relative abundance of ARB and selection for phenotypic resistance to sulfamethoxazole and ciprofloxacin than no addition of BAC. This finding is relevant because sulfamethoxazole and ciprofloxacin are currently used to treat a variety of bacterial infections. For example, sulfamethoxazole is used to treat cases of pneumonia and patients with human immunodeficiency virus (Thomas and Limper, 2014). In addition, BAC-fed bioreactors have been shown to select for the opportunistic pathogen Pseudomonas aeruginosa and select for ARB (Kim et al., 2018). While the data shown here make it clear that BAC will not universally select for antibiotic resistance to all antibiotics, the selection for resistance to ciprofloxacin and sulfamethoxazole is a potential concern and warrants further study.

Exposure to BAC also led to decreased resistance to other antibiotics (Fig. 2b). Exposure to $0.1 \mu \mathrm{g} \mathrm{L}^{-1}$ BAC decreased phenotypic resistance to ampicillin, streptomycin, and rifampicin by day 3. Over time, there were also observed increases in phenotypic resistance in the control community without exposure to BAC for ampicillin, chloramphenicol, streptomycin, rifampicin, trimethoprim, and cephalexin that did not occur when exposed to $0.1 \mu \mathrm{g} \mathrm{L}^{-1} \mathrm{BAC}$. For the control community, the relative abundance of ARB increased by day 3 for all six antibiotics listed $(p<0.043)$ and increased by days 7 and 14 for rifampicin, trimethoprim, and cephalexin $(p<0.048)$. No increases in relative abundance were observed over time for these six antibiotics when the community was treated with $0.1 \mu \mathrm{g} \mathrm{L}^{-1}$ BAC ( $p>0.056)$. Therefore, addition of BAC at $0.1 \mu \mathrm{g} \mathrm{L}-1$ concentration suppressed these increased phenotypic resistance trends. With no BAC added, there were significant increases in relative abundance of ARB that were no longer present when BAC was added to the community. BAC clearly affects antibiotic resistance profiles of bacteria, but not in the same way for all antibiotics. In this case, BAC increased selection for bacteria resistant to sulfamethoxazole and ciprofloxacin, and decreased selection for bacteria resistant to ampicillin, chloramphenicol, streptomycin, rifampicin, trimethoprim, and cephalexin. Phenotypic resistance to erythromycin increased on all days in both the communities exposed to BAC at $0.1 \mu \mathrm{g} \mathrm{L} \mathrm{L}^{-1}$ and the communities not exposed to BAC.

At $10 \mu \mathrm{g} \mathrm{L}^{-1} \mathrm{BAC}$, increases in phenotypic resistance to sulfamethoxazole, ciprofloxacin, and streptomycin were observed that did not occur in the control community (Fig. 2c). Relative abundance of bacteria resistant to both sulfamethoxazole and streptomycin increased on days 3, 7, and 14 when exposed to $10 \mu \mathrm{L} \mathrm{L}^{-1} \mathrm{BAC}(\mathrm{p}<0.015)$. The increased phenotypic resistance profiles to sulfamethoxazole were the same for the $10 \mu \mathrm{g} \mathrm{L}-1$ BAC treatment condition as they were for the $0.1 \mu \mathrm{g} \mathrm{L} \mathrm{L}^{-1} \mathrm{BAC}$ treatment condition. Exposure to $10 \mu \mathrm{g} \mathrm{L}^{-1} \mathrm{BAC}$ also increased phenotypic resistance to ciprofloxacin on day 3 and day $14(p<0.036)$. ARB in the control community demonstrated increased resistance to 
sulfamethoxazole and streptomycin by day $3(p<0.016)$ and were not statistically different on day 7 and day 14 ( $p>0.242)$. There were no increases in relative abundance of ARB to ciprofloxacin in the control community. The addition of BAC at $10 \mu \mathrm{g} \mathrm{L}^{-1}$ BAC increased selection of resistance to all three antibiotics by day 3 and day 7 . This finding is relevant because streptomycin is currently used to treat a variety of bacterial infections, including tuberculosis (Sotgiu et al., 2015). Tuberculosis is the second leading cause of death from an infectious disease worldwide, and is a very important antibiotic for protection of public health especially since many tuberculosis strains can acquire multi-drug resistances (Pham et al., 2015).

Exposure to BAC at $10 \mu \mathrm{g} \mathrm{L}^{-1}$ decreased phenotypic resistance to ampicillin, chloramphenicol, rifampicin, and trimethoprim. Similar to trends with $0.1 \mu \mathrm{g} \mathrm{L}{ }^{-1} \mathrm{BAC}$ exposure, statistical increases over time of relative abundance of ARB resistant to ampicillin, chloramphenicol, rifampicin, and trimethoprim were present with no BAC $(p<0.048)$. These increases were not observed for the $10 \mu \mathrm{g} \mathrm{L}^{-1} \mathrm{BAC}$ treatment condition. Exposure to BAC did not impact phenotypic resistance to erythromycin or cephalexin since there were increases on all three days present in both communities $(p<0.002)$.

Relative abundance of ARB resistant to tetracycline showed significant decreases over time in the control community with no BAC by day 3 and day $7(p<0.033)$ and a significant increase on day 14 $(p<0.001)$. However, these decreases were not present in the community exposed to $10 \mu \mathrm{g} \mathrm{L}^{-1} \mathrm{BAC}$ ( $p>0.583$ ). Thus, the addition of BAC allowed the community to maintain resistance to tetracycline by day 14 and eliminated decreases by day 3 and day 7 .

Exposure to $500 \mu \mathrm{g} \mathrm{L} \mathrm{L}^{-1} \mathrm{BAC}$ resulted in the same trend as 0.1 and $10 \mu \mathrm{g} \mathrm{L}$, i.e. phenotypic resistance to sulfamethoxazole and ciprofloxacin increased (Fig. 2d). Relative abundance of ARB resistant to both sulfamethoxazole and ciprofloxacin increased on days 3 and 7 when exposed to $500 \mu \mathrm{L} \mathrm{L}^{-1} \mathrm{BAC}$ $(p<0.019)$. In the control community, the relative abundance of ARB resistant to sulfamethoxazole increased by day $3(p=0.005)$. The increased phenotypic resistance profiles to sulfamethoxazole on day 3 and day 7 were the same for the $500 \mu \mathrm{g} \mathrm{L}-1$ BAC treatment condition as they were for the 0.1 and $10 \mu \mathrm{g} \mathrm{L}{ }^{-1} \mathrm{BAC}$ treatment conditions. There were no significant increases in phenotypic resistance to ciprofloxacin over time in the control community with no BAC. For the $500 \mu \mathrm{g} \mathrm{L}{ }^{-1} \mathrm{BAC}$ treatment condition, increased phenotypic resistance to ciprofloxacin was observed at day 3 and day 7 $(p<0.018)$. The increased phenotypic resistance profiles to ciprofloxacin on day 3 were the same for

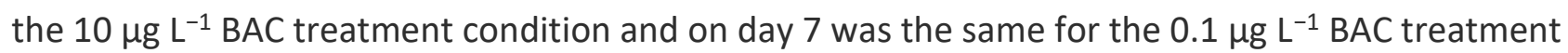
condition. The addition of BAC at $500 \mu \mathrm{g} \mathrm{L}^{-1}$ in the community positively selected for phenotypic resistance to both sulfamethoxazole and ciprofloxacin.

Exposure to BAC at $500 \mu \mathrm{g} \mathrm{L^{-1 }}$ decreased phenotypic resistance to chloramphenicol, streptomycin, rifampicin, trimethoprim, and tetracycline. Similar to trends with 0.1 and $10 \mu \mathrm{g} \mathrm{L}^{-1} \mathrm{BAC}$ exposure, statistical increases over time of relative abundance of ARB resistant to all five antibiotics listed were present in the control community $(p<0.048)$. These increases, mostly on day 3 , were not present with the $500 \mu \mathrm{g} \mathrm{L}^{-1} \mathrm{BAC}$ treatment condition. Following the same trend as $10 \mu \mathrm{g} \mathrm{L}^{-1} \mathrm{BAC}$, at $500 \mu \mathrm{g} \mathrm{L}^{-1} \mathrm{BAC}$ treatment did not impact phenotypic resistance to erythromycin or cephalexin since there was a statistical increase all three days present in both control and treated communities $(p<0.012)$. 
Furthermore, $500 \mu \mathrm{g} \mathrm{L}^{-1} \mathrm{BAC}$ treatment did not impact phenotypic resistance to ampicillin since there was a statistical increase by day 3 present in both communities $(p<0.043)$.

Relative abundance of ARB resistant to chloramphenicol and tetracycline significantly decreased over time in the control community by day $7(p<0.002)$. However, these decreases were not present in the community exposed to $500 \mu \mathrm{g} \mathrm{L}^{-1} \mathrm{BAC}(\mathrm{p}>0.956)$. Thus, exposure to BAC altered the community antibiotic resistance profiles and eliminated decreases in relative abundance in ARB to chloramphenicol and tetracycline by day 7 .

\subsection{Impact of BAC on bacterial genotypic selection for antibiotic resistance}

No statistically significant increases in ARGs compared to $0 \mu \mathrm{g} \mathrm{L}^{-1}$ BAC treatment were observed via qPCR on day 14 ( $p>0.05$ ) (Fig. 3). When compared to the initial day 0 source water sample, however, relative abundance of sul 1 and bla Interestingly, relative abundance of sul 1 increased significantly from day 0 to day 14 when exposed to 10 and $500 \mu \mathrm{g} \mathrm{L}^{-1}$ BAC ( $\left.p=0.011\right)$ and did not significantly increase in the no treatment control.

Similarly, relative abundance of bla TEM increased significantly from day 0 to day 14 when exposed to $500 \mu \mathrm{g} \mathrm{L}{ }^{-1} \mathrm{BAC}(\mathrm{p}=0.030)$ and did not significantly increase in the no treatment control. This result is relevant since the sul1 gene confers resistance to sulfonamides, synthetic antimicrobial agents (Gao et al., 2012; Heuer et al., 2011; Pruden et al., 2006), and the blaTEM gene confers resistance to $\beta$ lactams which is represented in numerous antibiotics and is a major public health concern (Livermore, 1995; Poole, 2004). 

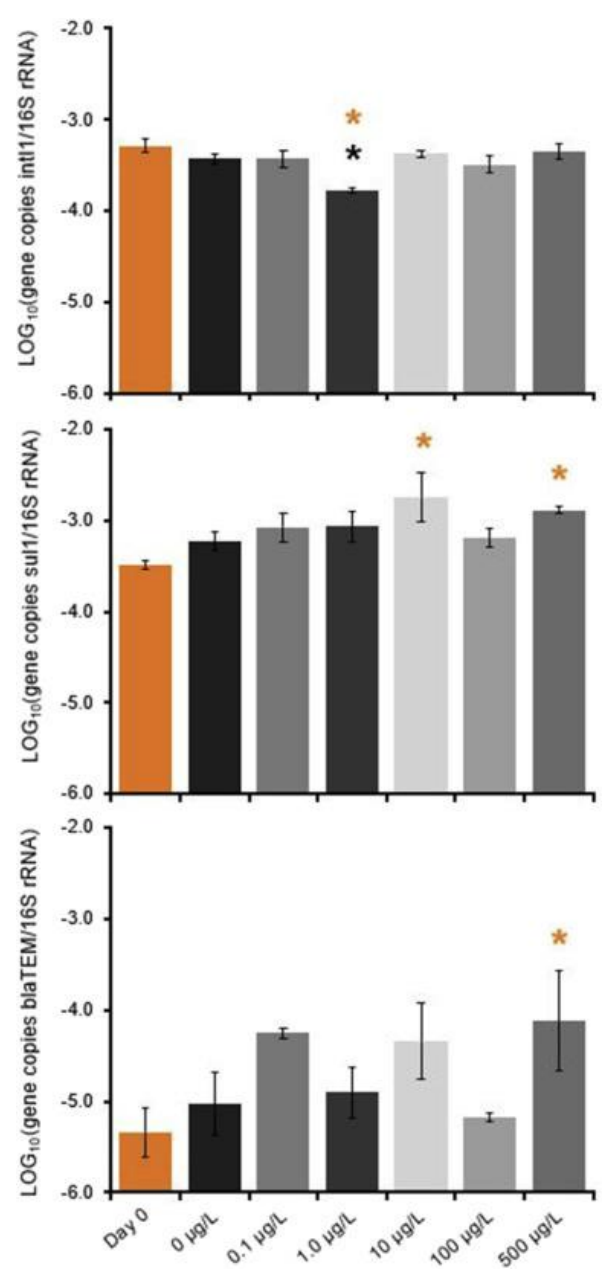

Fig. 3. The effect of BAC on relative abundance of the genes intl1, sul1, and bla $a_{\mathrm{TEM}}$. Bars represent average logscaled relative gene copy values relative to total $16 \mathrm{~S}$ rRNA gene copies. A higher bar signifies more relative gene copies and a greater quantity of resistance genes in the given sample. Error bars represent standard deviation of triplicate $\mathrm{qPCR}$ assays for each microcosm sample $(n=3)$. A black $*$ denotes statistical significance $(p<0.05)$ compared to control of $0 \mu \mathrm{g} \mathrm{L}{ }^{-1}$ BAC. Each BAC concentration from 0 to $500 \mu \mathrm{g} \mathrm{L}^{-1}$ was harvested at day 14 after exposure to the BAC treatment. An orange * denotes statistical significance $(p<0.05)$ compared to initial time control at day 0 . (For interpretation of the references to color in this figure legend, the reader is referred to the Web version of this article.)

Relative abundance gives insight into acquired resistance. Absolute abundances gives information into the total quantity of gene copies per liter of sample water which can be relevant when calculating risk. Absolute abundances of all genes are provided in Supplemental Fig. 3. The absolute abundance of sul1 significantly increased for all concentrations of BAC compared to day 0 . The absolute abundance of bla $a_{\text {TEM }}$ significantly increased compared to day 0 when exposed to BAC at $0.1 \mu g \mathrm{~L}^{-1}$ and $500 \mu \mathrm{g} \mathrm{L} \mathrm{L}^{-1}$.

When exposed to BAC treatment conditions, resistance to antibiotics was observed in viable cells phenotypically and showed different resistance trends with a genotypic approach over time. Acquired resistance is better to detect phenotypically because it indicates actual resistance is conferred. In addition, phenotypic resistance is medically relevant since it shows culturable results from bacteria that acquired antibiotic resistance as a result of BAC exposure. Culture-dependent techniques are 
valuable because they reveal resistance that may be difficult to quantify using molecular techniques such as qPCR. A limitation to culturable methods is that only a fraction of a bacterial community can be cultured. Both methods are necessary to tell a holistic story as molecular methods indicate broader potential for acquisition of antibiotic resistance. Molecular methods, such as qPCR, are required to detect resistance determinants in the community that could be relevant to public health in strains not accounted for by heterotrophic culture-dependent methods (Boolchandani et al., 2019). A limitation to this study is that metagenomics and metatranscriptomics were not conducted to quantify changes in total known ARG quantities and active expression of genes. Future work on this topic should use RNA to detect active expression of antibiotic resistance, including metatranscriptomics.

\section{Environmental implications}

Due to the recent ban on triclosan and triclocarban, replacement with BAC in consumer products warrants further study. As demonstrated in this study, BAC could lead to potential shifts in antibiotic resistance profiles in the environment. This study indicates that BAC alters resistance profiles to nine medically relevant antibiotics including ampicillin, chloramphenicol, sulfamethoxazole, streptomycin, rifampicin, ciprofloxacin, trimethoprim, tetracycline, and cephalexin. BAC positively selected for bacteria resistant to sulfamethoxazole and ciprofloxacin at three concentrations of BAC. The relative abundance of ARB resistant to sulfamethoxazole and ciprofloxacin significantly increased when BAC was present. This trend was not present in the control community with no BAC. This finding is important since exposure to BAC has led to cross-resistance with fluoroquinolones, the antibiotic class in which ciprofloxacin belongs (Jolivet-Gougeon et al., 2015). BAC also selected for antibiotic resistance genes sul1 and bla $a_{\text {TEM }}$ in exposed communities over time. Conversely, BAC negatively selected for bacteria resistant to ampicillin, streptomycin, and rifampicin at $0.1 \mu \mathrm{g} \mathrm{L}^{-1} \mathrm{BAC}$, and negatively selected for bacteria resistant to erythromycin, trimethoprim, and tetracycline at $500 \mu \mathrm{g} \mathrm{L}{ }^{-1} \mathrm{BAC}$. It should be noted that BAC has potential to limit the proliferation of antibiotic resistance to certain antibiotics in the environment at the specific concentrations and conditions tested. Since BAC can both positively and negatively select for ARB in the environment, further research is necessary to determine the impact of BAC on surface waters, especially if the concentration of BAC in environmental waters increases. BAC has recently been used as an alternative to both triclosan and triclocarban as an antimicrobial agent in consumer products. Both triclosan and triclocarban have been linked to the proliferation of antibiotic resistance in wastewater treatment processes (Carey et al., 2016a,b; Carey and McNamara, 2015; Carey and McNamara, 2016; Liu and Han, 2008; McClellan and Halden, 2010; McMurry et al., 1998). BAC also has the potential to select for antibiotic resistance in other environments, including wastewater biosolids, and important biological wastewater treatment processes such as activated sludge and anaerobic digestion (Baquero et al., 2008; Clara et al. 2007a, b). It is clear that BAC has the potential to alter antibiotic resistance profiles, specifically phenotypic resistance. Culture-dependent techniques are valuable for the ability to detect changes in phenotypic resistance in addition to potential for resistance observed from genotypic differences over time. Further study on the impact of BAC on antibiotic resistance is necessary to fully understand mechanisms of resistance in the environment and active expression of resistance genes.

\section{Declaration of competing interest}

There are no conflicts of interest to declare. 


\section{Acknowledgements}

K.H. was funded by Marquette University through the Water Quality Center. A.K. was funded by Marquette University through the GHR Foundation and the Earl B. and Charlotte Nelson Faculty Scholars Fund. The authors acknowledge the use of the LightCycler 96 from Marquette University, funded by the GHR Foundation.

Appendix A. Supplementary data

Help

The following is the Supplementary data to this article: Download : Download Word document (91KB) Multimedia component 1.

Download : Download XML file (260B)

Multimedia component 2.

Research data for this article

Data not available / Data will be made available on request

About research data

\section{References}

Baquero et al., 2008. Fernando Baquero, José Luis Martínez, Rafael Cantón. Antibiotics and antibiotic resistance in water environments. Curr. Opin. Biotechnol., 19 (3) (2008), pp. 260-265

Barber, 2019. Gerard R. Barber. The antibiotic paradox: how miracle drugs are destroying the miracle. Am. J. Health Syst. Pharm., 50 (8) (2019), pp. 1768-1771

Berendonk et al., 2015. Thomas U. Berendonk, Célia M. Manaia, Christophe Merlin, Despo FattaKassinos, Eddie Cytryn, Fiona Walsh, Helmut Bürgmann, Henning Sørum, Madelaine Norström, Marie Noëlle Pons, Norbert Kreuzinger, Pentti Huovinen, Stefania Stefani, Thomas Schwartz, Veljo Kisa nd, Fernando Baquero, José Luis Martinez. Tackling antibiotic resistance: the environmental framework. Nat. Rev. Microbiol., 13 (5) (2015), pp. 310-317

Boolchandani et al., 2019. Manish Boolchandani, Alaric W. D’Souza, Gautam Dantas. Sequencing-based methods and resources to study antimicrobial resistance. Nat. Rev. Genet., 20 (6) (2019), pp. 356-370

Braoudaki and Hilton, 2004. M. Braoudaki, A.C. Hilton. Adaptive resistance to biocides in Salmonella enterica and Escherichia coli 0157 and cross-resistance to antimicrobial agents. J. Clin. Microbiol., 42 (1) (2004), pp. 73-78

Carey and McNamara, 2015. D.E. Carey, P.J. McNamara. The impact of triclosan on the spread of antibiotic resistance in the environment. Front. Microbiol., 5 (JAN) (2015), pp. 1-11

Carey and McNamara, 2016. Daniel E. Carey, Patrick J. McNamara. Altered antibiotic tolerance in anaerobic digesters acclimated to triclosan or triclocarban. Chemosphere, 163 (2016), pp. 2226

Carey et al., 2016a. Daniel E. Carey, Daniel H. Zitomer, Krassimira R. Hristova, Anthony D. Kappell, Patrick J. McNamara. Triclocarban influences antibiotic resistance and alters 
anaerobic digester microbial community structure. Environ. Sci. Technol., 50 (1) (2016), pp. 126-134

Carey et al., 2016b. Daniel E. Carey, Daniel H. Zitomer, Anthony D. Kappell, Melinda J. Choi, Krassimira

R. Hristova, Patrick J. McNamara. Chronic exposure to triclosan Sustains microbial community shifts and alters antibiotic resistance gene levels in anaerobic digesters. Environ. Sci.:

Processes Impacts, 18 (8) (2016), pp. 1060-1067

Clara et al., 2007a. M. Clara, S. Scharf, C. Scheffknecht, O. Gans. Occurrence of selected surfactants in

untreated and treated sewage. Water Res., 41 (19) (2007), pp. 4339-4348

Clara et al., 2007b. M. Clara, S. Scharf, C. Scheffknecht, O. Gans. Occurrence of selected surfactants in

untreated and treated sewage. Water Res., 41 (19) (2007), pp. 4339-4348

CLSI, 2018. CLSI. Methods for Dilution Antimicrobial Susceptibility Tests for Bacteria That Grow

Aerobically. CLSI standard M07 (11th), Clinical and Laboratory Standards Institute, Wayne, PA (2018)

Dutta et al., 1993. P. Dutta, R. Rasaily, M.R. Saha, U. Mitra, S.K. Bhattacharya, M.K. Bhattacharya.

Ciprofloxacin for treatment of severe typhoid fever in children. Antimicrob. Agents

Chemother., 37 (5) (1993), pp. 1197-1199

Ferreccio et al., 2019. Catterine Ferreccio, J. Glenn .

orris Jr., Carmen Valdivieso, Ingeborg Prenzel, Viviana Sotomayor, George L. Drusano, Myron

M. Levine. Source the, infectious diseases, and No. Efficacy of Ciprofloxacin in the Treatment of Chronic Typhoid Carriers Published By, vol. 157, Oxford University Press Stable (Jun. 2019), pp. 1235-1239. 6. https://www.jstor.Org/Stable/30135753

Gadea et al., 2017. Rebeca Gadea, Miguel Ángel Fernández Fuentes, Rubén

Pérez Pulido, Antonio Gálvez, Elena Ortega. Effects of exposure to quaternary-ammonium-

based biocides on antimicrobial susceptibility and tolerance to physical stresses in bacteria from organic foods. Food Microbiol., 63 (2017), pp. 58-71

Gao et al., 2012. Panpan Gao, Daqing Mao, Yi Luo, Limei Wang, Bingjie Xu, Lin Xu. Occurrence of sulfonamide and tetracycline-resistant bacteria and resistance genes in aquaculture environment. Water Res., 46 (7) (2012), pp. 2355-2364

Garner et al., 2018. Emily Garner, Chaoqi Chen, Xia Kang, Jolene Bowers, David

M. Engelthaler, Jean McLain, Marc A. Edwards, Pruden Amy. Metagenomic characterization of antibiotic resistance genes in full-scale reclaimed water distribution systems and corresponding potable systems. Environ. Sci. Technol., 52 (11) (2018), pp. 6113-6125

Gillings et al., 2008.

Michael Gillings, Yan Boucher, Maurizio Labbate, Andrew Holmes, Samyuktha Krishnan, Marita Holley, H.W. Stokes. The evolution of class 1 integrons and the rise of antibiotic resistance.

J. Bacteriol., 190 (14) (2008), pp. 5095-5100

Gillings et al., 2015. Michael R. Gillings, William H. Gaze, Pruden Amy, Kornelia Smalla, James

M. Tiedje, Yong Guan Zhu. Using the class 1 integron-integrase gene as a proxy for anthropogenic pollution. ISME J., 9 (6) (2015), pp. 1269-1279

Gothwal and Shashidhar, 2015. Ritu Gothwal, Thhatikkonda Shashidhar. Antibiotic pollution in the environment: a review. Clean. - Soil, Air, Water, 43 (4) (2015), pp. 479-489

Heuer et al., 2011. Holger Heuer, Qodiah Solehati, Ute Zimmerling, Kristina Kleineidam, Michael Schloter, Tanja Müller, Andreas Focks, Sören Thiele-Bruhn, Kornelia Smalla. Accumulation of 
sulfonamide resistance genes in arable soils due to repeated application of manure containing sulfadiazine. Appl. Environ. Microbiol., 77 (7) (2011), pp. 2527-2530

Jolivet-Gougeon et al., 2015. Jolivet-Gougeon, Jean-Yves Maillard Anne, Martine BonnaureMallet, Pierre Tattevin, Sylvie Buffet-Bataillon. Efflux pump induction by quaternary ammonium compounds and fluoroquinolone resistance in bacteria. Future Microbiol., 11 (1) (2015), pp. 81-92

Joynson et al., 2002. J.A. Joynson, B. Forbes, R.J.W. Lambert. Adaptive resistance to benzalkonium chloride, amikacin and tobramycin: the effect on susceptibility to other antimicrobials. J. Appl. Microbiol., 93 (1) (2002), pp. 96-107

Jutkina et al., 2018. J. Jutkina, N.P. Marathe, C.F. Flach, D.G.J. Larsson. Antibiotics and common antibacterial biocides stimulate horizontal transfer of resistance at low concentrations. Sci. Total Environ., 616-617 (2018), pp. 172-178

Kappell et al., 2019. Anthony D. Kappell, Katherine R. Harrison, Patrick J. McNamara. Effects of zinc orthophosphate on the antibiotic resistant bacterial community of a source water used for drinking water treatment. Environ. Sci.: Water Res. Technol. (2019)

Kim et al., 2018. Minjae Kim, Michael R. Weigand, Seungdae Oh, Janet K. Hatt, Raj Krishnan, Ulas Tezel, Spyros G. Pavlostathis, Konstantinos T. Konstantinidis. Widely used benzalkonium chloride disinfectants can promote antibiotic resistance. Appl. Environ. Microbiol., 84 (17) (2018), pp. 1-14

Lambert et al., 2001. R.J.W. Lambert, J. Joynson, B. Forbes. The relationships and susceptibilities of some industrial, laboratory and clinical isolates of Pseudomonas aeruginosa to some antibiotics and biocides. J. Appl. Microbiol., 91 (6) (2001), pp. 972-984

LaPara et al., 2011. T.M. LaPara, T.R. Burch, P.J. McNamara, D.T. Tan, M. Yan, J.J. Eichmiller. Tertiarytreated municipal wastewater is a significant point source of antibiotic resistance genes into duluth-superior harbor. Environ. Sci. Technol., 45 (22) (2011), pp. 9543-9549

Liu and Han, 2008. Xinwang Liu, Shilian Han. Orness and parameterized RIM quantifier aggregation with OWA operators: a summary. Int. J. Approx. Reason., 48 (1) (2008), pp. 77-97

Livermore, 1995. D.M. Livermore. Beta-lactamases in laboratory and clinical resistance. Clin. Microbiol. Rev., 8 (4) (1995), pp. 557-584

Loughlin et al., 2002. M.F. Loughlin, M.V. Jones, P.A. Lambert. Pseudomonas aeruginosa cells adapted to benzalkonium chloride show resistance to other membrane-active agents but not to clinically relevant antibiotics. J. Antimicrob. Chemother., 49 (4) (2002), pp. 631-639

Martínez-Carballo et al., 2007. Elena Martínez-Carballo, Andrea Sitka, Carmen GonzálezBarreiro, Norbert Kreuzinger, Maria Fürhacker, Sigrid Scharf, Oliver Gans. Determination of selected quaternary ammonium compounds by liquid chromatography with mass spectrometry. Part I. Application to surface, waste and indirect discharge water samples in Austria. Environ. Pollut., 145 (2) (2007), pp. 489-496

McClellan and Halden, 2010. Kristin McClellan, Rolf U. Halden. Pharmaceuticals and personal care products in archived U.S. Biosolids from the 2001 EPA national sewage sludge survey. Water Res., 44 (2) (2010), pp. 658-668

McMurry et al., 1998. Laura M. McMurry, Margret Oethinger, Stuart B. Levy. Triclosan targets lipid synthesis [4]. Nature, 394 (6693) (1998), pp. 531-532 
McNamara and Levy, 2016. Patrick J. McNamara, Stuart B. Levy. Triclosan: an instructive tale. Antimicrob. Agents Chemother., 60 (12) (2016), pp. 02105-02116

McNamara et al., 2014. Patrick J. McNamara, Timothy M. Lapara, Paige J. Novak. The impacts of triclosan on anaerobic community structures, function, and antimicrobial resistance. Environ. Sci. Technol., 48 (13) (2014), pp. 7393-7400

Murray et al., 2019. Aimee K. Murray, Lihong Zhang, Jason Snape, William H. Gaze. Comparing the selective and Co-selective effects of different antimicrobials in bacterial communities. Int. J. Antimicrob. Agents, 53 (6) (2019), pp. 767-773

Nagy et al., 2015. Elisabeth Nagy, Edit Urb, Zsuzsa Eitel, Gabriella Terhes. Anaerobe investigation of the MICs of fi daxomicin and other antibiotics against Hungarian Clostridium dif fi cile isolates. Zsef S O, 31 (2015), pp. 47-49

O’Neill, 2016. Jim O'Neill. Tackling Drug-Resistant Infections Globally: Final Report and Recommendations', Review on Antimicrobial Resistance, Chaired by Jim O’Neill. (2016) https://Amr-Review.Org

Pal et al., 2015. Chandan Pal, Johan Bengtsson-Palme, Erik Kristiansson, D.G. Joaki, Larsson. Cooccurrence of resistance genes to antibiotics, biocides and metals reveals novel insights into their Co-selection potential. BMC Genomics, 16 (1) (2015)

Pham et al., 2015. Dinh Duy Pham, Elias Fattal, Nicolas Tsapis. Pulmonary drug delivery systems for tuberculosis treatment. Int. J. Pharm., 478 (2) (2015), pp. 517-529

Poole, 2004. K. Poole. Resistance to $\boldsymbol{\beta}$-lactam antibiotics. Cell. Mol. Life Sci., 61 (17) (2004), pp. $2200-$ 2223

Pruden et al., 2006. Amy Pruden, Ruoting Pei, Heather Storteboom, H. Kenneth, Carlson. Antibiotic resistance genes as emerging contaminants: studies in northern Colorado. Environ. Sci. Technol., 40 (23) (2006), pp. 7445-7450

Sotgiu et al., 2015. G. Sotgiu, R. Centis, L. D’Ambrosio, G. Battista Migliori. Tuberculosis treatment and drug regimens. Cold Spring Harbor Perspect. Med., 5 (5) (2015)

Tandukar et al., 2013. Madan Tandukar, Seungdae Oh, Ulas Tezel, Konstantinos T. Konstantinidis, Spyros G. Pavlostathis. Long-term exposure to benzalkonium chloride disinfectants results in change of microbial community structure and increased antimicrobial resistance. Environ. Sci. Technol., 47 (17) (2013), pp. 9730-9738

Team R Development Core, 2011. Team R Development Core. R: A Language and Environment for Statistical Computing. (2011)

Tezel and Pavlostathis, 2015. Ulas Tezel, Spyros G. Pavlostathis. Quaternary ammonium disinfectants: microbial Adaptation,Degradation and ecology. Curr. Opin. Biotechnol., 33 (Mic) (2015), pp. 296-304

Thomas and Limper, 2014. Charles F. Thomas, Andrew H. Limper. Treatment and Prevention of Pneumocystis Pneumonia in Non-HIV-infected Patients Treatment and Prevention of Pneumocystis Pneumonia in Non-HIV-infected Patients Page 2 of 14, vol. 1 (2014), pp. 1-14 US Dept. of Health and Human Services, 2013. US Dept. of Health and Human Services. Antibiotic Resistance Threats in the United States. vol. 2013 (2013)

Wang et al., 2012. Hong Wang, Sheldon Masters, Yanjuan Hong, Jonathan Stallings, Joseph O. Falkinham, Marc A. Edwards, Pruden Amy. Effect of disinfectant, water age, and pipe 
material on occurrence and persistence of Legionella, mycobacteria, Pseudomonas aeruginosa, and two amoebas. Environ. Sci. Technol., 46 (21) (2012), pp. 11566-11574

Watkinson et al., 2007. A.J. Watkinson, G.R. Micalizzi, J.R. Bates, S.D. Costanzo. Novel method for rapid assessment of antibiotic resistance in Escherichia coli isolates from environmental waters by use of a modified chromogenic agar. 73 (7) (2007), pp. 2224-2229

Wilkinson et al., 2019. John Wilkinson, Alistair Boxall, Kolpin Dana. A novel method to characterise levels of pharmaceutical pollution in large-scale Aquatic monitoring campaigns. Appl. Sci., 9 (7) (2019), p. 1368

World Health Organization, 2015. World Health Organization. Global Action Plan on Antimicrobial Resistance. World Health Organization (2015)

Xi et al., 2009. Chuanwu Xi, Yongli Zhang, Carl

F. Marrs, Ye Wen, Carl Simon, Betsy Foxman, Jerome Nriagu. Prevalence of antibiotic resistance in drinking water treatment and distribution systems. Appl. Environ. Microbiol., 75 (17) (2009), pp. 5714-5718

Xu et al., 2016. Like Xu, Weiying Ouyang, Yanyun Qian, Chao Su, Jianqiang Su, Hong Chen. Highthroughput profiling of antibiotic resistance genes in drinking water treatment plants and distribution systems. Environ. Pollut., 213 (2016), pp. 119-126

Zhang et al., 2015. Chang Zhang, Fang Cui, Guang ming Zeng, Min Jiang, Zhong zhu Yang, gang Yu Zhi, Meng ying Zhu, Liu qing Shen. Quaternary ammonium compounds (QACs): a review on occurrence, fate and toxicity in the environment. Sci. Total Environ., 518-519 (2015), pp. 352362

Zhang et al., 2017. Ye Zhang, April Z. Gu, Miao He, Dan Li, Jianmin Chen. Subinhibitory concentrations of disinfectants promote the horizontal transfer of multidrug resistance genes within and across genera. Environ. Sci. Technol., 51 (1) (2017), pp. 570-580

Zhang et al., 2018. Ye Zhang, April Z. Gu, Tianyu Cen, Xiangyang Li, Miao He, Dan Li, Jianmin Chen. Subinhibitory concentrations of heavy metals facilitate the horizontal transfer of plasmidmediated antibiotic resistance genes in water environment. Environ. Pollut., 237 (2018), pp. 74-82 